\title{
Positive solutions to boundary value problems of $p$-Laplacian with fractional derivative
}

\section{Xiaoyu Dong ${ }^{1}$, Zhanbing Bai ${ }^{*}$ (iD and Shuqin Zhang ${ }^{2}$}

${ }^{*}$ Correspondence:
zhanbingbai@163.com
${ }^{1}$ College of Mathematics and
System Science, Shandong
University of Science and
Technology, Qianwangang Road,
266590 Qingdao, P.R. China
Full list of author information is
available at the end of the article

\section{*Correspondence:}

${ }^{1}$ College of Mathematics and

System Science, Shandong

Technology, Qianwangang Road,

Ful 6590 Qingdao, P.R. China

available at the end of the article

\begin{abstract}
In this article, we consider the following boundary value problem of nonlinear fractional differential equation with $p$-Laplacian operator:

$$
\begin{aligned}
& D^{\alpha}\left(\phi_{p}\left(D^{\alpha} u(t)\right)\right)=f(t, u(t)), \quad 0<t<1, \\
& u(0)=u(1)=D^{\alpha} u(0)=D^{\alpha} u(1)=0,
\end{aligned}
$$

where $1<\alpha \leq 2$ is a real number, $D^{\alpha}$ is the conformable fractional derivative, $\phi_{p}(s)=|s|^{p-2} s, p>1, \phi_{p}^{-1}=\phi_{q}, 1 / p+1 / q=1$, and $f:[0,1] \times[0,+\infty) \rightarrow[0,+\infty)$ is continuous. One of the difficulties here is that the corresponding Green's function $G(t, s)$ is singular at $s=0$. By the use of an approximation method and fixed point theorems on cone, some existence and multiplicity results of positive solutions are acquired. Some examples are presented to illustrate the main results.
\end{abstract}

MSC: 34B18; 35J05; 34A08

Keywords: conformable fractional derivative; singular Green's function; fixed point theorems on cone; $p$-Laplacian operator

\section{Introduction}

Recently, differential equations have been of great interest. Integer order differential equations with $p$-Laplacian have been subject to a lot of research $[1,2]$. Now, many people pay attention to the existence and multiplicity of solutions for boundary value problems of fractional differential equations with $p$-Laplacian by the use of techniques of nonlinear analysis [3-6], upper and lower solutions method [7, 8], coincidence degree [9], Banach contraction mapping principle [10], etc.

Chen et al. [9] investigated the boundary value problem for a fractional differential equation with a $p$-Laplacian operator at resonance,

$$
\begin{aligned}
& D_{0+}^{\beta}\left(\phi_{p}\left(D_{0+}^{\alpha} u(t)\right)\right)=f\left(t, u(t), D_{0+}^{\alpha} u(t)\right), \quad 0<t<1, \\
& D_{0+}^{\alpha} u(0)=D_{0_{+}}^{\alpha} u(1)=0,
\end{aligned}
$$


where $0<\alpha, \beta \leq 1,1<\alpha+\beta \leq 2$, and $D_{0+}^{\alpha}$ is the Caputo fractional derivative. $\phi_{p}(s)=|s|^{p-2} s$, $p>1, \phi_{p}^{-1}=\phi_{q}, 1 / p+1 / q=1$. By using the coincidence degree theory, a new result of the existence of solution is obtained.

Lu et al. [7] studied the following $p$-Laplacian fractional differential equations boundary problems:

$$
\begin{aligned}
& D_{0+}^{\beta}\left(\phi_{p}\left(D_{0+}^{\alpha} u(t)\right)\right)=f(t, u(t)), \quad 0<t<1, \\
& u(0)=u^{\prime}(0)=u^{\prime}(1)=0, \quad D_{0+}^{\alpha} u(0)=D_{0+}^{\alpha} u(1)=0,
\end{aligned}
$$

where $2<\alpha \leq 3,1<\beta \leq 2, D_{0_{+}}^{\alpha}, D_{0_{+}}^{\beta}$ are the standard Riemann-Liouville fractional derivatives, $\phi_{p}(s)=|s|^{p-2} s, p>1, \phi_{p}^{-1}=\phi_{q}, 1 / p+1 / q=1$, and $f:[0,1] \times[0,+\infty) \rightarrow[0,+\infty)$ is continuous. By the properties of the Green's function, the Guo-Krasnosel'skii fixed point theorem, the Leggett-Williams fixed point theorem, and the upper and lower solutions method, some new existence results are obtained.

To the best of our knowledge, most of the literature about boundary value problems did not involve the singularity of $G(t, s)$. Therefore, in order to fill this gap in the literature, in this paper, we investigate the following $p$-Laplacian fractional differential equation boundary value problem:

$$
\begin{aligned}
& D^{\alpha}\left(\phi_{p}\left(D^{\alpha} u(t)\right)\right)=f(t, u(t)), \quad 0<t<1, \\
& u(0)=u(1)=D^{\alpha} u(0)=D^{\alpha} u(1)=0,
\end{aligned}
$$

where $1<\alpha \leq 2$ is a real number, $D^{\alpha}$ is the conformable fractional derivative, $\phi_{p}(s)=|s|^{p-2} s$, $p>1, \phi_{p}^{-1}=\phi_{q}, 1 / p+1 / q=1, f:[0,1] \times[0,+\infty) \rightarrow[0,+\infty)$ is continuous. By the approximation method and fixed point theorems on cone, some existence and multiplicity results of positive solutions are obtained. For $\alpha=2$, Problem (1.1), (1.2) is called a fourth $p$-Laplacian boundary value problem, which has been studied in [11-13].

The rest of this paper is organized as follows. In Section 2, we recall some concepts relative to the new conformable fractional calculus and give some lemmas with respect to the corresponding Green's function. In Section 3, we investigate the existence of positive solution for the boundary value problem (1.1), (1.2). In Section 4, the multiplicity of positive solutions is studied. In Section 5, we present some examples to illustrate our main results in Section 3 and Section 4, respectively.

\section{Preliminaries and lemmas}

For the convenience of the reader, we give some background material from fractional calculus theory to facilitate the analysis of Problem (1.1), (1.2). These results can be found in the recent literature; see [14-16].

Definition 2.1 Let $\alpha \in(n, n+1]$ and $f$ be a $n$-differentiable function at $t>0$, then the fractional conformable derivative of order $\alpha$ at $t>0$ is given by

$$
D^{\alpha} f(t)=D^{\alpha-n} f^{(n)}(t)=\lim _{\epsilon \rightarrow 0} \frac{f^{(n)}\left(t+\epsilon t^{n+1-\alpha}\right)-f^{(n)}(t)}{\epsilon},
$$


provided the limit of the right hand side exists. If $f$ is $\alpha$-differentiable in some $(0, a), a>0$, and $\lim _{t \rightarrow 0^{+}} D^{\alpha} f(t)$ exists, then define

$$
D^{\alpha} f(0)=\lim _{t \rightarrow 0^{+}} D^{\alpha} f(t)
$$

Remark 2.1 As a basic example, given $\alpha \in(n, n+1]$, we have

$$
D^{\alpha} t^{k}=0
$$

where $k=0,1, \ldots, n$.

Lemma 2.1 Let $t>0, \alpha \in(n, n+1]$. The function $f(t)$ is $(n+1)$-differentiable if and only if $f$ is $\alpha$-differentiable, moreover, $D^{\alpha} f(t)=t^{n+1-\alpha} f^{(n+1)}(t)$.

Proof Let $h=\epsilon t^{n+1-\alpha}+O\left(\epsilon^{2}\right)$. With Definition 2.1, we have

$$
\begin{aligned}
D^{\alpha} f(t) & =\lim _{\epsilon \rightarrow 0} \frac{f^{(n)}\left(t+\epsilon t^{n+1-\alpha}\right)-f^{(n)}(t)}{\epsilon} \\
& =\lim _{\epsilon \rightarrow 0} \frac{f^{(n)}(t+h)-f^{(n)}(t)}{\frac{h}{t^{n+1-\alpha}+O(\epsilon)}} \\
& =t^{n+1-\alpha} f^{(n+1)}(t) .
\end{aligned}
$$

The proof is complete.

Definition 2.2 ([14]) Let $\alpha \in(n, n+1]$. The fractional integral of order $\alpha>0$ at $t>0$ of a function $f:(0, \infty) \rightarrow R$ is given by

$$
I^{\alpha} f(t)=I^{n+1}\left(t^{\alpha-n-1} f(t)\right)=\frac{1}{n !} \int_{0}^{t}(t-s)^{n} s^{\alpha-n-1} f(s) d s,
$$

where $I^{n+1}$ denotes the integration operator of order $n+1$.

Lemma 2.2 Let $\alpha \in(n, n+1]$ and $f$ be a continuous function defined in $(0,+\infty)$, one has $D^{\alpha} I^{\alpha} f(t)=f(t)$ for $t>0$.

Proof Since $f(t)$ is continuous, $I^{\alpha} f(t)$ is $(n+1)$-differentiable. In view of Lemma 2.1 one has

$$
\begin{aligned}
D^{\alpha}\left(I^{\alpha} f\right)(t) & =t^{n+1-\alpha} \frac{d^{n+1}}{d t^{n+1}}\left(\frac{1}{n !} \int_{0}^{t}(t-s)^{n} s^{\alpha-n-1} f(s) d s\right) \\
& =t^{n+1-\alpha} f(t) t^{\alpha-n-1} \\
& =f(t) .
\end{aligned}
$$

The proof is complete.

Lemma 2.3 ([16] Mean value theorem) Let $a \geq 0$ and $f:[a, b] \rightarrow R$ be a function with the properties that 
(1) $f$ is continuous on $[a, b]$,

(2) $f$ is $\alpha$-differentiable on $(a, b)$ for some $\alpha \in(0,1)$.

Then there exists $c \in(a, b)$ such that

$$
D^{\alpha} f(c)=\frac{f(b)-f(a)}{\frac{1}{\alpha} b^{\alpha}-\frac{1}{\alpha} a^{\alpha}} .
$$

Lemma 2.4 Let $\alpha \in(n, n+1], f$ be a $\alpha$-differentiable function at $t>0$, then $D^{\alpha} f(t)=0$ for $t \in(0, \infty)$ if and only if $f(t)=a_{0}+a_{1} t+\cdots+a_{n-1} t^{n-1}+a_{n} t^{n}$, where $a_{k} \in R$, for $k=0,1, \ldots, n$.

Proof The sufficiency follows by Remark 2.1.

Next, given $t_{1}, t_{2} \in(0, \infty)$ with $t_{1}<t_{2}$, by Lemma 2.3 , there exists $\xi \in\left(t_{1}, t_{2}\right)$ such that

$$
f^{(n)}\left(t_{2}\right)-f^{(n)}\left(t_{1}\right)=D^{\alpha} f(\xi)\left(\frac{1}{\alpha} t_{2}^{\alpha}-\frac{1}{\alpha} t_{1}^{\alpha}\right)
$$

By means of $D^{\alpha} f(\xi)=0$, we have $f^{(n)}\left(t_{2}\right)=f^{(n)}\left(t_{1}\right)$, with arbitrary $t_{1}, t_{2}$, one has $f^{(n)}(t) \equiv C$ and $f(t)=a_{0}+a_{1} t+\cdots+a_{n-1} t^{n-1}+a_{n} t^{n}$, for $t \in(0, \infty)$.

With Lemma 2.2 and Lemma 2.4, the following lemma is immediate.

Lemma 2.5 Assume that $u \in C(0,+\infty)$ with a fractional derivative of order $\alpha \in(n, n+1]$ that belongs to $C(0,1) \cap L(0,1)$. Then

$$
I^{\alpha} D^{\alpha} u(t)=u(t)+c_{0}+c_{1} t+\cdots+c_{n} t^{n}
$$

for some $c_{k} \in R, k=0,1, \ldots, n$.

Now, we present the Green's function. In the following arguments, we always suppose that $\alpha \in(1,2]$.

Lemma 2.6 Given $y \in C[0,1]$, the unique solution of

$$
\begin{aligned}
& D^{\alpha} u(t)+y(t)=0, \quad 0<t<1, \\
& u(0)=u(1)=0,
\end{aligned}
$$

is

$$
u(t)=\int_{0}^{1} G(t, s) y(s) d s
$$

where

$$
G(t, s)= \begin{cases}(1-t) s^{\alpha-1}, & \text { for } 0 \leq s \leq t \leq 1 \\ t s^{\alpha-2}(1-s), & \text { for } 0 \leq t \leq s \leq 1\end{cases}
$$


Proof Applying Lemma 2.5 we reduce equation (2.5) to an equivalent integral equation,

$$
\begin{aligned}
u(t) & =-I^{\alpha} y(t)+c_{0}+c_{1} t \\
& =-\int_{0}^{t}(t-s) s^{\alpha-2} y(s) d s+c_{0}+c_{1} t,
\end{aligned}
$$

for some $c_{0}, c_{1} \in R$. By (2.6), we have $c_{0}=0, c_{1}=\int_{0}^{1}(t-s) s^{\alpha-2} y(s) d s$. Therefore, the unique solution of Problem (2.5), (2.6) is

$$
\begin{aligned}
u(t)= & -\int_{0}^{t}(t-s) s^{\alpha-2} y(s) d s+t \int_{0}^{1}(t-s) s^{\alpha-2} y(s) d s \\
= & \int_{0}^{t}\left(-t s^{\alpha-2}+s^{\alpha-1}+t s^{\alpha-2}-t s^{\alpha-1}\right) y(s) d s \\
& +\int_{t}^{1}\left(t s^{\alpha-2}-t s^{\alpha-1}\right) y(s) d s \\
= & \int_{0}^{t}(1-t) s^{\alpha-1} y(s) d s+\int_{t}^{1} t s^{\alpha-2}(1-s) y(s) d s \\
= & \int_{0}^{1} G(t, s) y(s) d s .
\end{aligned}
$$

The proof is complete.

We point out here that (2.7) becomes the usual Green's function when $\alpha=2$.

Lemma 2.7 Let $y \in C[0,1]$ and $1<\alpha \leq 2$. Then the fractional differential equation boundary value problem

$$
\begin{aligned}
& D^{\alpha}\left(\phi_{p}\left(D^{\alpha} u(t)\right)\right)=y(t), \quad 0<t<1, \\
& u(0)=u(1)=D^{\alpha} u(0)=D^{\alpha} u(1)=0,
\end{aligned}
$$

has a unique solution,

$$
u(t)=\int_{0}^{1} G(t, s) \phi_{q}\left(\int_{0}^{1} G(s, \tau) y(\tau) d \tau\right) d s
$$

Proof Apply the operator $I^{\alpha}$ on both sides of (2.8), with Lemma 2.5, we have

$$
\phi_{p}\left(D^{\alpha} u(t)\right)+C_{0}+C_{1} t=I^{\alpha} y(t) .
$$

So,

$$
\begin{aligned}
\phi_{p}\left(D^{\alpha} u(t)\right) & =I^{\alpha} y(t)-C_{0}-C_{1} t \\
& =\int_{0}^{t}(t-\tau) \tau^{\alpha-2} y(\tau) d \tau-C_{0}-C_{1} t
\end{aligned}
$$


for some $C_{0}, C_{1} \in \mathbb{R}$. By the boundary conditions $D^{\alpha} u(0)=D^{\alpha} u(1)=0$, we have

$$
C_{0}=0, \quad C_{1}=\int_{0}^{1}(1-\tau) \tau^{\alpha-2} y(\tau) d \tau
$$

Therefore, the solution $u(t)$ of the fractional differential equation boundary value problem (2.8) and (2.9) satisfies

$$
\begin{aligned}
\phi_{p}\left(D^{\alpha} u(t)\right) & =\int_{0}^{t}(t-\tau) \tau^{\alpha-2} y(\tau) d \tau-t \int_{0}^{1}(1-\tau) \tau^{\alpha-2} y(\tau) d \tau \\
& =-\int_{0}^{1} G(t, \tau) y(\tau) d \tau .
\end{aligned}
$$

Thus, the fractional differential equation boundary value problem (2.8) and (2.9) is equivalent to the problem

$$
\begin{aligned}
& D^{\alpha} u(t)+\phi_{q}\left(\int_{0}^{1} G(t, \tau) y(\tau) d \tau\right)=0, \quad 0<t<1, \\
& u(0)=u(1)=0 .
\end{aligned}
$$

Lemma 2.6 implies that the fractional differential equation boundary value problem (2.8) and (2.9) has a unique solution,

$$
u(t)=\int_{0}^{1} G(t, s) \phi_{q}\left(\int_{0}^{1} G(s, \tau) y(\tau) d \tau\right) d s .
$$

The proof is complete.

Lemma 2.8 The function $G(t, s)$ defined by (2.7) satisfies the following properties:

(i) $G(t, s)>0$, for all $t, s \in(0,1)$;

(ii) $\min _{\frac{1}{4} \leq t \leq \frac{3}{4}} G(t, s) \geq \frac{1}{4} \max _{0 \leq t \leq 1} G(t, s)=\frac{1}{4} G(s, s)$, for $s \in(0,1)$.

Proof Observing the expression of $G(t, s)$, it is clear that $G(t, s)>0$ for $t, s \in(0,1)$. Next, for given $s \in(0,1)$ we consider the partial derivative of $G(t, s)$ with respect to $t$,

$$
\frac{\partial G(t, s)}{\partial t}= \begin{cases}-s^{\alpha-1}, & s \leq t \\ s^{\alpha-2}(1-s), & t \leq s\end{cases}
$$

This shows that $G(t, s)$ is decreasing with respect to $t$ for $s \leq t$, and increasing for $t \leq s$.

So,

$$
\max _{0 \leq t \leq 1} G(t, s)=G(s, s)=(1-s) s^{\alpha-1}
$$

and

$$
\min _{\frac{1}{4} \leq t \leq \frac{3}{4}} G(t, s)= \begin{cases}\frac{1}{4} s^{\alpha-1}, & s \in\left(0, \frac{1}{2}\right] ; \\ \frac{1}{4}(1-s) s^{\alpha-2}, & s \in\left[\frac{1}{2}, 1\right) .\end{cases}
$$


Let

$$
\gamma(s)=\frac{\min _{\frac{1}{4} \leq t \leq \frac{3}{4}} G(t, s)}{G(s, s)}= \begin{cases}\frac{1}{4(1-s)}, & s \in\left(0, \frac{1}{2}\right] ; \\ \frac{1}{4 s}, & s \in\left[\frac{1}{2}, 1\right) .\end{cases}
$$

Clearly, $\gamma(s) \geq \frac{1}{4}, s \in(0,1)$, the proof is complete.

It should be noted that the constant bound is new for fractional derivatives. It was pointed out that the Riemann-Liouville fractional derivative does not allow one to get a positive constant boundary (see [3], Remark 2.2).

Lemma 2.9 ([17])

(1) If $1<q \leq 2$, then

$$
\left|\phi_{q}(u+v)-\phi_{q}(u)\right| \leq 2^{2-q}|v|^{q-1}
$$

for all $u, v \in R$.

(2) If $q>2$, then

$$
\left|\phi_{q}(u+v)-\phi_{q}(u)\right| \leq(q-1)(|u|+|v|)^{q-2}|v|
$$

for all $u, v \in R$.

Lemma 2.10 ([18]) Suppose $E$ is a Banach space and $T_{n}: E \rightarrow E, n=3,4, \ldots$ are completely continuous operators, $T: E \rightarrow E$. If $\left\|T_{n} u-T u\right\|$ uniformly converges to zero when $n \rightarrow \infty$ for all bounded set $\Omega \subseteq E$, then $T: E \rightarrow E$ is completely continuous.

Definition 2.3 The map $\theta$ is said to be a nonnegative continuous concave functional on a cone $P$ of a Banach space $E$ provided that $\theta: P \rightarrow[0, \infty)$ is continuous and

$$
\theta(t x+(1-t) y) \geq t \theta(x)+(1-t) \theta(y)
$$

for all $x, y \in P$ and $0<t<1$.

The following fixed point theorems are useful in our proofs.

Lemma 2.11 ([19]) Let $E$ be a Banach space, $P \subseteq E$ a cone, and $\Omega_{1}, \Omega_{2}$ two bounded open balls of $E$ centered at the origin with $\overline{\Omega_{1}} \subset \Omega_{2}$. Suppose that $\mathcal{A}: P \cap\left(\overline{\Omega_{2}} \backslash \Omega_{1}\right) \rightarrow P$ is a completely continuous operator such that either

(i) $\|\mathcal{A} x\| \leq\|x\|, x \in P \cap \partial \Omega_{1}$, and $\|\mathcal{A} x\| \geq\|x\|, x \in P \cap \partial \Omega_{2}$ or

(ii) $\|\mathcal{A} x\| \geq\|x\|, x \in P \cap \partial \Omega_{1}$, and $\|\mathcal{A} x\| \leq\|x\|, x \in P \cap \partial \Omega_{2}$

holds. Then $\mathcal{A}$ has a fixed point in $P \cap\left(\overline{\Omega_{2}} \backslash \Omega_{1}\right)$.

Lemma 2.12 ([20]) Let $P$ be a cone in a real Banach space $E, P_{c}=\{x \in P \mid\|x\| \leq c\}, \theta$ a nonnegative continuous concave functional on $P$ such that $\theta(x) \leq\|x\|$, for all $x \in \overline{P_{c}}$, and $P(\theta, b, d)=\{x \in P \mid b \leq \theta(x),\|x\| \leq d\}$. Suppose $\mathcal{A}: \overline{P_{c}} \rightarrow \overline{P_{c}}$ is completely continuous and there exist constants $0<a<b<d \leq c$ such that 
(C1) $\{x \in P(\theta, b, d) \mid \theta(x)>b\}$ is non-empty, and $\theta(\mathcal{A} x)>b$, for $x \in P(\theta, b, d)$;

(C2) $\|\mathcal{A} x\|<a$, for $x \leq a$;

(C3) $\theta(\mathcal{A} x)>b$, for $x \in P(\theta, b, c)$ with $\|\mathcal{A} x\|>d$.

Then $\mathcal{A}$ has at least three fixed points $x_{1}, x_{2}, x_{3}$ with

$$
\left\|x_{1}\right\|<a, \quad b<\theta\left(x_{2}\right), \quad a<\left\|x_{3}\right\|, \quad \theta\left(x_{3}\right)<b .
$$

Remark 2.2 ([20]) If we have $d=c$, then condition (C1) of Lemma 2.12 implies condition (C3) of Lemma 2.12.

\section{Existence results}

Let $E=C[0,1]$ be endowed with the ordering $u \leq v$ if $u(t) \leq v(t)$ for all $t \in[0,1]$, and the maximum norm $\|u\|=\max _{0 \leq t \leq 1}|u(t)|$. Define

$$
P=\left\{u \in E \mid u(t) \geq 0, \min _{\frac{1}{4} \leq t \leq \frac{3}{4}} u(t) \geq \frac{1}{4}\|u\|\right\} .
$$

Define the nonnegative continuous concave functional $\theta$ by

$$
\theta(u)=\min _{\frac{1}{4} \leq t \leq \frac{3}{4}}|u(t)| .
$$

Given the continuous function $f \in C([0,1] \times[0, \infty))$, define $T, T_{n}: P \rightarrow E$ as

$$
\begin{aligned}
& (T u)(t):=\int_{0}^{1} G(t, s) \phi_{q}\left(\int_{0}^{1} G(s, \tau) f(\tau, u(\tau)) d \tau\right) d s \\
& \left(T_{n} u\right)(t):=\int_{\frac{1}{n}}^{1} G(t, s) \phi_{q}\left(\int_{\frac{1}{n}}^{1} G(s, \tau) f(\tau, u(\tau)) d \tau\right) d s, \quad n=3,4, \ldots .
\end{aligned}
$$

Lemma 3.1 $T: P \rightarrow P$ is completely continuous.

Proof Firstly, we show that $T_{n}: P \rightarrow P$ are completely continuous for $n=3,4, \ldots$ Given $u \in P$, with Lemma 2.8 and the nonnegativity of $f(t, u)$, one has

$$
\begin{aligned}
\left(T_{n} u\right)(t) & =\int_{\frac{1}{n}}^{1} G(t, s) \phi_{q}\left(\int_{\frac{1}{n}}^{1} G(s, \tau) f(\tau, u(\tau)) d \tau\right) d s \\
& \leq \int_{\frac{1}{n}}^{1} G(s, s) \phi_{q}\left(\int_{\frac{1}{n}}^{1} G(s, \tau) f(\tau, u(\tau)) d \tau\right) d s
\end{aligned}
$$

so

$$
\left\|T_{n} u\right\| \leq \int_{\frac{1}{n}}^{1} G(s, s) \phi_{q}\left(\int_{\frac{1}{n}}^{1} G(s, \tau) f(s, u(s)) d \tau\right) d s .
$$


And next, if $u \in P$,

$$
\begin{aligned}
\min _{\frac{1}{4} \leq t \leq \frac{3}{4}}\left(T_{n} u\right)(t) & =\min _{\frac{1}{4} \leq t \leq \frac{3}{4}} \int_{\frac{1}{n}}^{1} G(t, s) \phi_{q}\left(\int_{\frac{1}{n}}^{1} G(s, \tau) f(s, u(s)) d \tau\right) d s \\
& \geq \frac{1}{4} \int_{\frac{1}{n}}^{1} G(s, s) \phi_{q}\left(\int_{\frac{1}{n}}^{1} G(s, \tau) f(s, u(s)) d \tau\right) d s \\
& \geq \frac{1}{4}\left\|T_{n} u\right\| .
\end{aligned}
$$

As a consequence $T_{n}: P \rightarrow P$. The continuity of $T_{n}$ follows by the continuity of $G(t, s)$ and $f(t, u)$. Let $\Omega \subset P$ be bounded, i.e., there exists a positive constant $M>0$ such that $\|u\| \leq M$ for all $u \in \Omega$. Let

$$
L=\max _{0 \leq t \leq 1,0 \leq u \leq M}|f(t, u)|+1, \quad H=\int_{0}^{1} G(s, s) d s+1,
$$

then, for $u \in \Omega$, we have

$$
\begin{aligned}
\left|\left(T_{n} u\right)(t)\right| & =\int_{\frac{1}{n}}^{1} G(t, s) \phi_{q}\left(\int_{\frac{1}{n}}^{1} G(s, \tau) f(s \tau, u(\tau)) d \tau\right) d s \\
& \leq L^{q-1} H^{q} \\
& <+\infty .
\end{aligned}
$$

Hence, $T_{n}(\Omega)$ is bounded for $n=3,4, \ldots$ On the other hand, given $\epsilon>0$, let

$$
\delta=\frac{\epsilon}{L^{q-1} H^{q-1}\left(\frac{1}{\alpha-1}+\frac{1}{\alpha}\right)},
$$

then, for each $u \in \Omega, t_{1}, t_{2} \in[0,1], t_{1}<t_{2}$, and $t_{2}-t_{1}<\delta$, one has

$$
\left|\left(T_{n} u\right)\left(t_{2}\right)-\left(T_{n} u\right)\left(t_{1}\right)\right|<\epsilon .
$$

That is to say that $T_{n}(\Omega)$ has equicontinuity. In fact, we consider three situations.

(1) $0<t_{1}<t_{2}<\frac{1}{n}$.

$$
\begin{aligned}
& \left|\left(T_{n} u\right)\left(t_{2}\right)-\left(T_{n} u\right)\left(t_{1}\right)\right| \\
& =\mid \int_{\frac{1}{n}}^{1} G\left(t_{2}, s\right) \phi_{q}\left(\int_{\frac{1}{n}}^{1} G(s, \tau) f(\tau, u(\tau)) d \tau\right) d s \\
& \quad-\int_{\frac{1}{n}}^{1} G\left(t_{1}, s\right) \phi_{q}\left(\int_{\frac{1}{n}}^{1} G(s, \tau) f(\tau, u(\tau)) d \tau\right) d s \mid \\
& \leq L^{q-1} H^{q-1} \int_{\frac{1}{n}}^{1}\left|G\left(t_{2}, s\right)-G\left(t_{1}, s\right)\right| d s \\
& =L^{q-1} H^{q-1} \int_{\frac{1}{n}}^{1}\left(t_{2}-t_{1}\right) s^{\alpha-2}(1-s) d s \\
& \quad \leq L^{q-1} H^{q-1}\left(t_{2}-t_{1}\right)\left(\frac{1}{\alpha-1}-\frac{1}{\alpha}\right)
\end{aligned}
$$

$<\epsilon$. 
(2) $0<t_{1}<\frac{1}{n}<t_{2}<1$.

$$
\begin{aligned}
\left|\left(T_{n} u\right)\left(t_{2}\right)-\left(T_{n} u\right)\left(t_{1}\right)\right| & \\
\leq & L^{q-1} H^{q-1}\left(\int_{\frac{1}{n}}^{t_{2}}\left|G\left(t_{2}, s\right)-G\left(t_{1}, s\right)\right| d s\right. \\
& \left.+\int_{t_{2}}^{1}\left|G\left(t_{2}, s\right)-G\left(t_{1}, s\right)\right| d s\right) \\
\leq & (L H)^{q-1}\left(\int_{\frac{1}{n}}^{t_{2}} s^{\alpha-2}\left[\left(s-t_{1}\right)+\left(t_{2}-t_{1}\right) s\right] d s\right. \\
& \left.+\int_{t_{2}}^{1}\left(t_{2}-t_{1}\right) s^{\alpha-2}(1-s) d s\right) \\
\leq & (L H)^{q-1}\left(t_{2}-t_{1}\right) \int_{0}^{1}\left(s^{\alpha-2}+s^{\alpha-1}\right) d s \\
\leq & (L H)^{q-1}\left(t_{2}-t_{1}\right)\left(\frac{1}{\alpha-1}+\frac{1}{\alpha}\right) \\
< & \epsilon
\end{aligned}
$$

(3) $\frac{1}{n}<t_{1}<t_{2}<1$.

$$
\begin{aligned}
\left|\left(T_{n} u\right)\left(t_{2}\right)-\left(T_{n} u\right)\left(t_{1}\right)\right| & \\
\leq & L^{q-1} H^{q-1}\left(\int_{\frac{1}{n}}^{t_{1}}\left|G\left(t_{2}, s\right)-G\left(t_{1}, s\right)\right| d s+\int_{t_{1}}^{t_{2}}\left|G\left(t_{2}, s\right)-G\left(t_{1}, s\right)\right| d s\right. \\
& \left.\quad+\int_{t_{2}}^{1}\left|G\left(t_{2}, s\right)-G\left(t_{1}, s\right)\right| d s\right) \\
= & L^{q-1} H^{q-1}\left(\int_{\frac{1}{n}}^{t_{1}}\left(t_{2}-t_{1}\right) s^{\alpha-1} d s+\int_{t_{1}}^{t_{2}}\left(t_{2}-t_{1}\right)\left(s^{\alpha-2}+s^{\alpha-1}\right) d s\right. \\
& \left.\quad+\int_{t_{2}}^{1}\left(t_{2}-t_{1}\right)\left(s^{\alpha-2}-s^{\alpha-1}\right) d s\right) \\
\leq & L^{q-1} H^{q-1}\left(t_{2}-t_{1}\right) \int_{0}^{1}\left(s^{\alpha-2}+s^{\alpha-1}\right) d s \\
\leq & L^{q-1} H^{q-1}\left(t_{2}-t_{1}\right)\left(\frac{1}{\alpha-1}+\frac{1}{\alpha}\right) \\
< & \epsilon .
\end{aligned}
$$

By the means of the Arzela-Ascoli theorem, we see that $T_{n}: P \rightarrow P$ are completely continuous operators.

Secondly, it is clear that $T: P \rightarrow P$. We prove that $T_{n}: P \rightarrow P$ uniformly converges to $T$ and $T: P \rightarrow P$ is completely continuous too.

With the use of Lemma 2.9, we have

$$
\phi_{q}(A+B)<\phi_{q}(A)+2 \phi_{q}(B)+(q-1)(A+B)^{q-2} B .
$$


Given $\epsilon>0$, let

$$
N=\left(\frac{\frac{2 L^{q-1} H}{\alpha^{q-1}}+\frac{q L^{q-1} H^{q-1}}{\alpha}}{\epsilon}\right),
$$

then $\left\|T_{n} u-T u\right\|<\epsilon$, for all $n>N$. In fact,

$$
\begin{aligned}
& \left\|T_{n} u-T u\right\| \\
& =\max _{0 \leq t \leq 1}\left|\left(T_{n} u\right)(t)-(T u)(t)\right| \\
& =\int_{0}^{1} G(t, s) \phi_{q}\left(\int_{0}^{1} G(s, \tau) f(\tau, u(\tau)) d \tau\right) d s \\
& -\int_{\frac{1}{n}}^{1} G(t, s) \phi_{q}\left(\int_{\frac{1}{n}}^{1} G(s, \tau) f(\tau, u(\tau)) d \tau\right) d s \\
& <\int_{0}^{1} G(t, s)\left[\phi_{q}\left(\int_{\frac{1}{n}}^{1} G(s, \tau) f(\tau, u(\tau)) d \tau\right)+2 \phi_{q}\left(\int_{0}^{\frac{1}{n}} G(s, \tau) f(\tau, u(\tau)) d \tau\right)\right. \\
& \left.+(q-1)\left(\int_{0}^{1} G(s, \tau) f(\tau, u(\tau)) d \tau\right)^{q-2} \int_{0}^{\frac{1}{n}} G(s, \tau) f(\tau, u(\tau)) d \tau\right] d s \\
& -\int_{\frac{1}{n}}^{1} G(t, s) \phi_{q}\left(\int_{\frac{1}{n}}^{1} G(s, \tau) f(s, u(s)) d \tau\right) d s \\
& \leq \int_{0}^{\frac{1}{n}} G(t, s) \phi_{q}\left(\int_{\frac{1}{n}}^{1} G(s, \tau) f(\tau, u(\tau)) d \tau\right) d s \\
& +2 L^{q-1} \int_{0}^{1} G(s, s) d s \phi_{q}\left(\int_{0}^{\frac{1}{n}} G(\tau, \tau) d \tau\right) \\
& +(q-1) L^{q-1} \int_{0}^{1} G(s, s) d s\left(\int_{0}^{1} G(\tau, \tau) d \tau\right)^{q-2} \int_{0}^{\frac{1}{n}} G(\tau, \tau) d \tau \\
& \leq\left(\frac{L^{q-1} H^{q-1}}{\alpha}+\frac{2 L^{q-1} H}{\alpha^{q-1}}+\frac{(q-1) L^{q-1} H^{q-1}}{\alpha}\right)\left(\frac{1}{n}\right)^{\alpha} \\
& =\left(\frac{2 L^{q-1} H}{\alpha^{q-1}}+\frac{q L^{q-1} H^{q-1}}{\alpha}\right)\left(\frac{1}{n}\right)^{\alpha} \\
& <\epsilon \text {. }
\end{aligned}
$$

By the use of Lemma 2.10, $T: P \rightarrow P$ is completely continuous.

Denote

$$
\begin{aligned}
& M=\left(\int_{0}^{1} G(s, s) d s \phi_{q}\left(\int_{0}^{1} G(\tau, \tau) d \tau\right)\right)^{-1}, \\
& N=\left(\int_{\frac{1}{4}}^{\frac{3}{4}} G(s, s) d s \phi_{q}\left(\int_{\frac{1}{4}}^{\frac{3}{4}} G(\tau, \tau) d \tau\right)\right)^{-1} .
\end{aligned}
$$

Theorem 3.1 Let $f(t, u)$ be continuous on $[0,1] \times[0, \infty)$. Assume that there exist two different positive constants $r_{2}, r_{1}$, and $r_{2} \neq r_{1}$ such that 
(H1) $f(t, u) \leq \phi_{p}\left(M r_{1}\right)$, for $(t, u) \in[0,1] \times\left[0, r_{1}\right]$;

(H2) $f(t, u) \geq \phi_{p}\left(N r_{2}\right)$, for $(t, u) \in\left[\frac{1}{4}, \frac{3}{4}\right] \times\left[\frac{1}{4} r_{2}, r_{2}\right]$.

Then Problem (1.1), (1.2) has at least one positive solution $u$ such that $\min \left\{r_{2}, r_{1}\right\} \leq\|u\| \leq$ $\max \left\{r_{2}, r_{1}\right\}$.

Proof By Lemma 3.1, $T: P \rightarrow P$ is completely continuous. Without loss of generality, suppose $0<r_{1}<r_{2}$, and let

$$
\Omega_{1}:=\left\{u \in P \mid\|u\|<r_{1}\right\}, \quad \Omega_{2}:=\left\{u \in P \mid\|u\|<r_{2}\right\} .
$$

For $u \in \partial \Omega_{1}$, we have $0 \leq u(t) \leq r_{1}$ for all $t \in[0,1]$. It follows from (H1) that

$$
\begin{aligned}
\|T u\| & =\max _{0 \leq t \leq 1}\left|\int_{0}^{1} G(t, s) \phi_{q}\left(\int_{0}^{1} G(s, \tau) f(\tau, u(\tau)) d \tau\right) d s\right| \\
& \leq M r_{1} \int_{0}^{1} G(s, s) d s \phi_{q}\left(\int_{0}^{1} G(\tau, \tau) d \tau\right)=r_{1}=\|u\| .
\end{aligned}
$$

So,

$\|T u\| \leq\|u\|, \quad$ for $u \in \partial \Omega_{1}$.

For $u \in \partial \Omega_{2}$, by the definition of $P$, we have

$$
u(t) \geq \frac{1}{4}\|u\|=\frac{1}{4} r_{2}, \quad t \in\left[\frac{1}{4}, \frac{3}{4}\right] .
$$

By assumption (H2), for $t \in\left[\frac{1}{4}, \frac{3}{4}\right]$, we have

$$
\begin{aligned}
(T u)(t) & =\int_{0}^{1} G(t, s) \phi_{q}\left(\int_{0}^{1} G(s, \tau) f(\tau, u(\tau)) d \tau\right) d s \\
& \geq \int_{0}^{1} \frac{1}{4} G(s, s) \phi_{q}\left(\int_{\frac{1}{4}}^{\frac{3}{4}} \frac{1}{4} G(\tau, \tau) f(\tau, u(\tau)) d \tau\right) d s \\
& \geq N r_{2} \int_{\frac{1}{4}}^{\frac{3}{4}} \frac{1}{4} G(s, s) d s \phi_{q}\left(\int_{\frac{1}{4}}^{\frac{3}{4}} \frac{1}{4} G(\tau, \tau) d \tau\right)=r_{2}=\|u\| .
\end{aligned}
$$

So,

$$
\|T u\| \geq\|u\|, \quad \text { for } u \in \partial \Omega_{2} .
$$

Therefore, by Lemma 2.11, we complete the proof.

\section{Multiplicity}

Theorem 4.1 Suppose $f(t, u)$ is continuous on $[0,1] \times[0, \infty)$ and there exist constants $0<a<\frac{1}{4} b$ such that the following assumptions hold:

(A1) $f(t, u) \leq \phi_{p}(M a)$, for $(t, u) \in[0,1] \times[0, a]$;

(A2) $f(t, u) \geq \phi_{p}\left(\frac{1}{4} N b\right)$, for $(t, u) \in\left[\frac{1}{4}, \frac{3}{4}\right] \times\left[\frac{1}{4} b, b\right]$; 
(A3) $f(t, u) \leq \phi_{p}(M b)$, for $(t, u) \in[0,1] \times[0, b]$.

Then the boundary value problem (1.1), (1.2) has at least three positive solutions $u_{1}, u_{2}, u_{3}$ with

$$
\begin{aligned}
& \max _{0 \leq t \leq 1}\left|u_{1}(t)\right|<a, \quad \frac{1}{4} b<\min _{\frac{1}{4} \leq t \leq \frac{3}{4}}\left|u_{2}(t)\right|<\max _{0 \leq t \leq 1}\left|u_{2}(t)\right| \leq b, \\
& a<\max _{0 \leq t \leq 1}\left|u_{3}(t)\right| \leq b, \quad \min _{\frac{1}{4} \leq t \leq \frac{3}{4}}\left|u_{3}(t)\right|<\frac{1}{4} b .
\end{aligned}
$$

Proof We show that all the conditions of Lemma 2.12 are satisfied. If $u \in \bar{P}_{b}$, then $\|u\| \leq b$. Assumption (A3) implies $f(t, u(t)) \leq M b$ for $0 \leq t \leq 1$, consequently,

$$
\begin{aligned}
\|T u\| & =\max _{0 \leq t \leq 1}\left|\int_{0}^{1} G(t, s) \phi_{q}\left(\int_{0}^{1} G(s, \tau) f(\tau, u(\tau)) d \tau\right) d s\right| \\
& \leq \int_{0}^{1} G(s, s) \phi_{q}\left(\int_{0}^{1} G(\tau, \tau) f(\tau, u(\tau)) d \tau\right) d s \\
& \leq M b \int_{0}^{1} G(s, s) d s \phi_{q}\left(\int_{0}^{1} G(\tau, \tau) d \tau\right) \leq b .
\end{aligned}
$$

Hence, $T: \bar{P}_{b} \rightarrow \bar{P}_{b}$. Similarly, if $u \in \bar{P}_{a}$, then assumption (A1) yields $f(t, u(t)) \leq M a, 0 \leq$ $t \leq 1$. Therefore, condition (C2) of Lemma 2.12 is satisfied.

Choose

$$
u(t)=\frac{\frac{1}{4} b+b}{2}=\frac{5 b}{8}, \quad 0 \leq t \leq 1
$$

Then $u(t) \in P\left(\theta, \frac{1}{4} b, b\right), \theta(u)=\theta\left(\frac{5 b}{8}\right)>\frac{1}{4} b$, consequently,

$$
\left\{u \in P\left(\theta, \frac{1}{4} b, b\right) \mid \theta(u)>\frac{1}{4} b\right\} \neq \emptyset .
$$

Hence, if $u \in P\left(\theta, \frac{1}{4} b, b\right)$, then $\frac{1}{4} b \leq u(t) \leq b$ for $\frac{1}{4} \leq t \leq \frac{3}{4}$. From assumption (A2), we have $f(t, u(t)) \geq N\left(\frac{1}{4} b\right)$ for $\frac{1}{4} \leq t \leq \frac{3}{4}$. So

$$
\begin{aligned}
\theta(T u) & =\min _{\frac{1}{4} \leq t \leq \frac{3}{4}}|(T u)(t)| \geq \int_{0}^{1} \frac{1}{4} G(s, s) \phi_{q}\left(\int_{\frac{1}{4}}^{\frac{3}{4}} \frac{1}{4} G(\tau, \tau) f(\tau, u(\tau)) d \tau\right) d s \\
& >N \frac{1}{4} b \int_{\frac{1}{4}}^{\frac{3}{4}} \frac{1}{4} G(s, s) d s \phi_{q}\left(\int_{\frac{1}{4}}^{\frac{3}{4}} \frac{1}{4} G(\tau, \tau) d \tau\right)=\frac{1}{4} b .
\end{aligned}
$$

That is,

$$
\theta(T u)>\frac{1}{4} b, \quad \text { for all } u \in P\left(\theta, \frac{1}{4} b, b\right) .
$$

This shows that condition (C1) of Lemma 2.12 is satisfied. 
By Lemma 2.12 and Remark 2.2, Problem (1.1), (1.2) has at least three positive solutions $u_{1}, u_{2}, u_{3}$, satisfying

$$
\begin{aligned}
& \max _{0 \leq t \leq 1}\left|u_{1}(t)\right|<a, \quad \frac{1}{4} b<\min _{\frac{1}{4} \leq t \leq \frac{3}{4}}\left|u_{2}(t)\right|, \\
& a<\max _{0 \leq t \leq 1}\left|u_{3}(t)\right|, \quad \min _{\frac{1}{4} \leq t \leq \frac{3}{4}}\left|u_{3}(t)\right|<\frac{1}{4} b .
\end{aligned}
$$

The proof is complete.

\section{Some examples}

Example 5.1 Consider the following boundary value problem:

$$
\begin{aligned}
& D^{\frac{3}{2}}\left(\phi_{2}\left(D^{\frac{3}{2}} u(t)\right)\right)=2+\sqrt{u}+\frac{t^{2}}{2}, \quad 0<t<1, \\
& u(0)=u(1)=D^{\frac{3}{2}} u(0)=D^{\frac{3}{2}} u(1)=0 .
\end{aligned}
$$

By a simple computation, we obtain $M=3.75, N \approx 5.987$. Choose $r_{1}=1, r_{2}=\frac{1}{3}$, then

$$
\begin{aligned}
& f(t, u)=2+\sqrt{u}+\frac{t^{2}}{2} \leq 3.5<\phi_{2}\left(M r_{1}\right)=3.75, \quad \text { for }(t, u) \in[0,1] \times[0,1] \\
& f(t, u)=2+\sqrt{u}+\frac{t^{2}}{2} \geq 2>\phi_{2}\left(N r_{2}\right) \approx 1.996, \quad \text { for }(t, u) \in\left[\frac{1}{4}, \frac{3}{4}\right] \times\left[\frac{1}{12}, \frac{1}{3}\right] .
\end{aligned}
$$

With the use of Theorem 3.1, the fractional differential equation boundary value problem (5.1) and (5.2) has at least one positive solution $u$ such that $\frac{1}{3} \leq\|u\| \leq 1$.

Example 5.2 Consider the following boundary value problem:

$$
\begin{aligned}
& D^{\frac{3}{2}}\left(\phi_{2}\left(D^{\frac{3}{2}} u(t)\right)\right)=f(t, u), \quad 0<t<1, \\
& u(0)=u(1)=D^{\frac{3}{2}} u(0)=D^{\frac{3}{2}} u(1)=0,
\end{aligned}
$$

where

$$
f(t, u)= \begin{cases}7 u^{3}+\frac{1}{3} t^{2}, & u \leq 1 \\ 6+u+\frac{19}{4} t^{2}, & u \geq 1\end{cases}
$$

We obtain $M=3.75, N \approx 5.987$. Choose $a=0.1, b=4$, then

$$
\begin{aligned}
& f(t, u)=7 u^{3}+\frac{t^{2}}{3}<0.344<\phi_{2}(M a)=0.375, \quad \text { for }(t, u) \in[0,1] \times[0,0.1] \\
& f(t, u)=6+u+\frac{19}{4} t^{2} \geq 6>\phi_{2}\left(\frac{N b}{4}\right) \approx 5.987, \quad \text { for }(t, u) \in\left[\frac{1}{4}, \frac{3}{4}\right] \times[1,4] \\
& f(t, u)=6+u+\frac{19}{4} t^{2} \leq 14.75<\phi_{2}(M b)=15, \quad \text { for }(t, u) \in[0,1] \times[0,4]
\end{aligned}
$$


With the use of Theorem 4.1, the fractional differential equation boundary value problem (5.3) and (5.4) has at least three positive solutions $u_{1}, u_{2}$, and $u_{3}$ with

$$
\begin{aligned}
& \max _{0 \leq t \leq 1}\left|u_{1}(t)\right|<0.1, \quad 1<\min _{\frac{1}{4} \leq t \leq \frac{3}{4}}\left|u_{2}(t)\right|<\max _{0 \leq t \leq 1}\left|u_{2}(t)\right| \leq 4, \\
& 0.1<\max _{0 \leq t \leq 1}\left|u_{3}(t)\right| \leq 4, \quad \min _{\frac{1}{4} \leq t \leq \frac{3}{4}}\left|u_{3}(t)\right|<1 .
\end{aligned}
$$

\section{Competing interests}

The authors declare that they have no competing interests.

\section{Authors' contributions}

All authors contributed equally to the writing of this paper. All authors read and approved the final manuscript.

\section{Author details}

${ }^{1}$ College of Mathematics and System Science, Shandong University of Science and Technology, Qianwangang Road, 266590 Qingdao, P.R. China. ${ }^{2}$ School of Science, China University of Mining and Technology, 10083 Beijing, P.R. China.

\section{Acknowledgements}

The authors express their sincere thanks to the anonymous reviews for their valuable suggestions and corrections for improving the quality of the paper. This work is supported by NSFC (11571207, 11371364), the Taishan Scholar project, and SDUST graduate innovation project SDKDYC170343.

Received: 17 September 2016 Accepted: 19 December 2016 Published online: 03 January 2017

\section{References}

1. Agarwal, RP, Lü, HS, O'Regan, D: Eigenvalues and the one-dimensional p-Laplacian. J. Math. Anal. Appl. 266, 383-400 (2002)

2. Avery, R, Henderson, J: Existence of three positive pseudo-symmetric solutions for and the one-dimensional p-Laplacian. J. Math. Anal. Appl. 277, 395-404 (2003)

3. Zhao, C, Cui, YJ: Monotone iterative technique for nonlinear four-order two-point boundary value problem. J. Shandong Univ. Sci. Technol. Nat. Sci. 35(6), 108-113 (2016) (in Chinese)

4. Han, ZL, Lu, HL, Sun, SS, Yang, DW: Positive solutions to boundary-value problems of p-Laplacian fractional differential equations with a parameter in the boundary. Electron. J. Differ. Equ. 2012, 213 (2012)

5. Liu, ZH, Lu, L: A class of BVPs for nonlinear fractional differential equations with p-Laplacian operator. Electro. J. Qual. Theo. Differ. Equ. 2012, 70 (2012)

6. Wang, J, Xiang, H, Liu, Z: Existence of concave positive solutions for boundary-value problem of nonlinear fractional differential equation with p-Laplacian operator. Int. J. Math. Math. Sci. 2010, Article ID 495138 (2010)

7. Lu, HL, Han, ZL, Sun, SR, Liu, J: Existence on positive solutions for boundary value problems of nonlinear fractional differential equations with p-Laplacian. Adv. Differ. Equ. 2013, 30 (2013)

8. Wang, J, Xiang, H: Upper and lower solutions method for a class of singular fractional boundary-value problems with p-Laplacian operator. Abstr. Appl. Anal. 2010, Article ID 971824 (2010)

9. Chen, T, Liu, W, Hu, ZG: A boundary value problem for fractional differential equation with $p$-Laplacian operator at resonance. Nonlinear Anal. 75, 3210-3217 (2012)

10. Liu, X, Jia, M, Xiang, X: On the solvability of a fractional differential equation model involving the $p$-Laplacian operator. Comput. Math. Appl. 64, 3267-3275 (2012)

11. Bai, ZB Zhang, S, Sun, SJ, Yin, C: Monotone iterative method for a class of fractional differential equations. Electro. J. Differ. Equ. 2016, 1-8 (2016)

12. $\mathrm{Wu}, \mathrm{HH}$, Sun, SJ: Multiple positive solutions for a fourth order boundary value via variational method. J. Shandong Univ. Sci. Technol. Nat. Sci. 33(2), 96-99 (2014) (in Chinese)

13. Zhang, XG, Liu, LS: Positive solutions of fourth-order four-point boundary value problems with p-Laplacian operator. J. Math. Anal. Appl. 336, 1414-1423 (2007)

14. Batarfi, H, Losada, J, Nieto, JJ, Shammakh, W: Three-point boundary value problems for conformable fractional differential equations. J. Funct. Spaces 205, 1-3 (2015)

15. Dong, X, Bai, Z, Zhang, W: Positive solutions for nonlinear eigenvalue problems with conformable fractional differential derivatives. J. Shandong Univ. Sci. Technol. Nat. Sci. 35(3), 85-91 (2016) (in Chinese)

16. Khalil, R, Al Horani, M, Yousef, A, Sababheh, M: A new definition of fractional derivative. J. Comput. Appl. Math. 264, 65-70 (2014)

17. Yan, P: Nonresonance for one-dimensional p-Laplacian with regular restoring. J. Math. Anal. Appl. 285, 141-154 (2003)

18. Guo, DJ: Nonlinear Functional Analysis. Shandong Science and Technology Publishing House, Jinan (2001) (in Chinese)

19. Krasnosel'skii, MA: Positive Solutions of Operator Equations. Noordhoff, Groningen (1964)

20. Leggett, RW, Williams, LR: Multiple positive fixed points of nonlinear operators on ordered Banach spaces. Indiana Univ. Math. J. 30, 637-688 (1979) 